\title{
The Impact of Heteromultivalency in Lectin Recognition and Glycosidase Inhibition: An Integrated Mechanistic Study
}

\author{
M. Isabel García-Moreno, ${ }^{[a]}$ Fernando Ortega-Caballero, ${ }^{[a]}$ Rocío Rísquez-Cuadro, ${ }^{[a]}$ Carmen Ortiz \\ Mellet, ${ }^{*[a]}$ José M. García Fernández ${ }^{*[b]}$
}

\begin{abstract}
The vision of multivalency as a strategy limited to achieve affinity enhancements between a protein receptor and its putative sugar ligand (glycotope) has proven too simplistic. On the one hand, binding of a glycotope in a dense glycocalix-like construct to a lectin partner has been shown to be sensitive to the presence of a third sugar entity (heterocluster effect). On the other hand, several carbohydrate processing enzymes (glycosidases and glycosyltransferases) have been found to be also responsive to multivalent presentations of binding partners (multivalent enzyme inhibition), a phenomenon first discovered for iminosugar-type inhibitory species (inhitopes) and recently demonstrated for multivalent carbohydrate constructs. By assessing a series of homo and heteroclusters combining $\alpha$-D-glucopyranosyl-related glycotopes and inhitopes, here we show that multivalency and heteromultivalency govern indeed both kinds of events, allowing activating, deactivating or enhancing specific recognition phenomena towards a spectrum of lectin and glycosidase partners in a multimodal manner. This unified scenario originates from the ability of (hetero)multivalent architectures to trigger glycosidase binding modes that are reminiscent of those harnessed by lectins, which should be considered when profiling the biological activity of multivalent architectures.
\end{abstract}

\section{Introduction}

The saccharidic portion in glycoconjugates is involved in different binding-recognition processes that trigger or inhibit a wide variety of biological phenomena, including cell-cell communication, host-pathogen interactions, immune response or cancer metastasis, acting as information storage and transmission devices. ${ }^{[1]}$ Although only a handful of monosaccharide subunits participate directly in the supramolecular events eliciting those responses, their multiple modes of combination results in an extremely versatile ensemble of readable arrangements that conforms the so-called "sugar code". [2] Carbohydrate recognizing (lectins) and processing (glycosidases, glycosyltransferases) proteins can act

[a] Dr. M. I. García-Moreno, Dr. F. Ortega-Caballero, Dr. RísquezCuadro, Prof. C. Ortiz Mellet

Department of Organic Chemistry, Faculty of Chemistry

University of Sevilla

c/ Profesor García González 1, 41012 Sevilla, Spain

E-mail: mellet@us.es

[b] Prof. J. M. García Fernández

Instituto de Investigaciones Químicas (IIQ)

CSIC - University of Sevilla

Avda. Americo Vespucio 49, 41092 Sevilla, Spain

E-mail: jogarcia@iiq.csic.es

Supporting information for this article is given via a link at the end of the document. as the complementary reader partners. The mechanism by which these two families of carbohydrate-interacting proteins interpret the information stored in the oligosaccharides is thought to be starkly different. Thus, glycosidases generally bind to single oligosaccharide sequences with high affinity and selectivity, which is followed by conformational distortion and enzymatic hydrolysis of the critical glycosidic linkage. ${ }^{[3]}$ On the contrary, many lectins operate through multivalent interactions, typical monovalent carbohydrate ligands binding only weakly. ${ }^{[4]}$ This dogma has largely dominated research on artificial glycosidase and lectin ligands through the last decades. Thus, many synthetic polyconjugates with various copies of an identical individual sugar recognition motif (glycotope) attached to a polymeric, dendritic or molecular scaffold have been developed aiming at mimicking and matching the arrangement of complementary lectin receptors in their natural mode of affinity enhancement. ${ }^{[5,6]}$ The design of glycosidase inhibitors has focused instead on monovalent glycomimetics with a resemblance to the natural substrate or to the corresponding transition state. ${ }^{[7]}$

The above doctrine has been seriously questioned by recent experimental evidences. On the one hand, it has been demonstrated that glycoheterogeneity has an impact on carbohydrate-protein recognition events, ${ }^{[8-12]}$ a concept that has been termed "the heterocluster effect" ${ }^{[13,14]}$ On the other hand, clusterization of a carbohydrate-processing-enzyme inhibitory moiety (inhitope), generally an iminosugar-type glycomimetic, has been shown to modulate and eventually amplify the ability of inhibiting certain glycosidases ${ }^{[15-20]}$ and glycosyltransferases, ${ }^{[21]}$ a counterintuitive phenomenon named "multivalent enzyme inhibition" or "multivalent inhibition effect". ${ }^{[22,23]}$ Similarly to the classical multivalent or cluster effect operating in homogeneous glycoligand-lectin interactions, the heterocluster and multivalent inhibition effects depend on the interacting partners and on architectural parameters. More recently, it was found that $\alpha-D-$ glucopyranoside and $\alpha$-D-mannopyranoside glycotopes, which are specific ligands of lectins such as the plant lectin concanavalin $\mathrm{A}$ (ConA), the uropathogenic Escherichia coli lectin Fim-H or the dendritic cell-specific C-type lectin DC-SIGN, ${ }^{[6]}$ become potent inhibitors of some glycosidases when (hetero)multivalently exposed at the surface of nanodiamonds (Figure $1 \mathrm{~A}$ ), definitely blurring the boundaries between lectin and glycosidase recognition. ${ }^{[24]}$ Further investigations using $\alpha$-Dmannopyranoside-, $\beta$-D-galactopyranoside-, $\alpha$-L-fucopyranosideand $\beta$-lactoside-fullerene conjugates (Figure $1 \mathrm{~B}$ ) supported the new paradigm, ${ }^{[25]}$ highlighting the urgent need for integrated studies on the consequences of (hetero)multivalency in the selectivity profile of glycotope/inhitope motifs not only towards one or more different lectins or one or more different glycosidases, but also towards a spectrum of lectin and glycosidase partners. 
A

$\alpha$-D-glucopyranoside

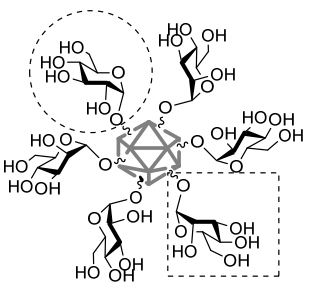

$\alpha$-D-mannopyranoside

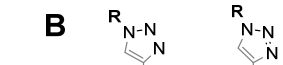

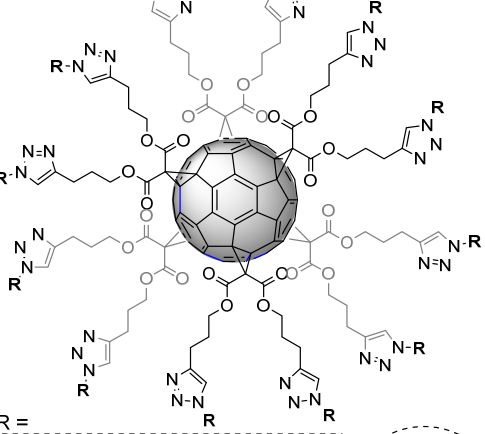

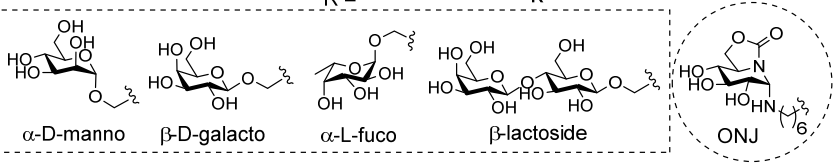

\section{Results and Discussion}

\section{Glyco(mimetic)cluster Library Design}

The facial anisotropy of the $\beta C D$ macroring and the availability of a variety of efficient selective chemical functionalization methodologies make $\beta C D$-scaffolded glycol(mimetic)clusters privileged architectures to assess the effect of the relative density and orientation of ligands in the interaction with complementary biomolecular partners. ${ }^{[27]}$ In order to get information on the effect of primary structure (i.e., the nature and number of copies of the ligands) and secondary structure (i.e., their spatial arrangement) modifications on the glycosidase and lectin recognition properties, the library of glyco(mimetic) clusters 1-12 was synthesized (Figure 2).

Figure 1. Schematic representation of heterovalent glyco-nanodiamonds (A) and homovalent glyco(mimetic)-fullerenes (B) showing dual lectin-glycosidase binding abilities.

We have previously reported that the $\mathrm{sp}^{2}$-iminosugar-type ${ }^{[26]}$ $5 \mathrm{~N}, 6 \mathrm{O}$-oxomethylidenenojirimycin ( $\mathrm{ONJ}$; Figure $1 \mathrm{~B})$ residue, a potent $\alpha$-glucosidase inhitope, is also a ligand of peanut (Arachis Hypogaea) agglutinin (PNA) lectin, enabling competitive assays that provide unique information on the binding modes elicited by multivalency. ${ }^{[17]}$ In principle, the strategy can be also applied to glycoclusters with lectin binding and glycosidase inhibitory properties and, by extension, to mixed inhitope/glycotope heteromultivalent constructs, offering an excellent opportunity to investigate the molecular basis of the effects triggered by (hetero)multivalency in both types of proteins. As a proof of concept, we have now synthesized a series of cyclomaltoheptose $(\beta$-cyclodextrin, $\quad \beta C D)$ conjugates incorporating ONJ and/or $\alpha$-D-glucopyranoside ( $\alpha$-Glc) moieties intentionally designed to pinpoint analogies/differences in recognition processes against lectins and glycosidases. First, we have evaluated the impact of (hetero)multivalency in PNA/ONJ and ConA $/ \alpha-$ Glc binding. Second, we determined the effect of (hetero)multivalency in the inhibition of maltase (yeast) isomaltase (yeast) and $\alpha$-mannosidase (Jack bean). The three are multivalency-sensitive enzymes, but whereas the catalytic site of $\alpha$-mannosidase is rather accessible and can host inhitope moieties at the periphery of multivalent inhibitors, maltase and isomaltase possesses deep catalytic sites that can hardly be access by ligands scaffolded in nanosized platforms. ${ }^{[17,25]}$ Lectin/lectin and lectin/glycosidase cross-linking experiments in the absence or presence of reference ligands have been additionally conducted to identify binding modes and provide a rational for the observed multimodal effect of (hetero)multivalency in lectin recognition and glycosidase inhibition. The ensemble of data incite the conceptualization of (hetero)multivalency as a tool to administer the information encoded in glyo(mimetic) devices in a much broader context that previously thought.

Figure 2. Structures of the glycotope ( $\alpha-G \mathrm{lc}) /$ inhitope (ONJ) conjugates prepared in this work and schematic representation of their different topologies $(\mathrm{A}-\mathrm{C})$. 
Conjugates 1-4 are monovalent controls; their comparative assessment should allow establishing the effect of the $\beta C D$ scaffold in the recognition of the glycotope $(\alpha-G l c)$ or inhitope (ONJ) motif by protein partners. Interrogation of the heptavalent (5-7; including the hemi-substituted derivative 8) and tetradecavalent (9-12) sublibraries will provide information on the influence of multivalency and heteromultivalency in a faceoriented, relatively compact arrangement (Figure 3, structure A) or in a quasi-globular distribution of the coating recognition elements (Figure 3, structures B and C), respectively. Note that whereas the heterovalent constructs 7 and 11 expose statistic ("shuffled") 1:1 distributions of the glycotope and inhitope moieties in separate branches, compound $\mathbf{1 2}$ is a single isomer derivative bearing a $\alpha-G \mid c$ and a ONJ motif in every arm; it was purposely incorporated in our study to evaluate the potential influence of orientational factors in the behavior of high-density heteroclusters against lectins and glycosidases.

\section{Impact of (hetero)multivalency in lectin binding}

The relative lectin binding abilities of compounds 1-12 against the lectins ConA and PNA were evaluated using a competitive enzyme-linked lectin assay (ELLA). ${ }^{[6]}$ Briefly, the fraction of horseradish-labelled lectin (HRP-ConA or HRP-PNA) bound to a microplate-supported reference ligand (yeast mannan or a galactose-coated glycopolymer, respectively) in the absence and presence of increasing concentrations of the new conjugates was monitored. The concentrations needed to achieve $50 \%$ inhibition of the reference ligand-lectin association $\left(\mathrm{IC}_{50}\right.$ values; assumed to be proportional to the corresponding binding energies) are collected in Table 1 . The $I C_{50}$ values obtained for methyl $\alpha$-D-mannopyranoside $(1050 \pm 50 \mu \mathrm{M})$ and methyl $\alpha$-D-glucopyranoside $(11000 \pm 100 \mu \mathrm{M})$ against ConA and for lactose against PNA $(1350 \pm 10 \mu \mathrm{M})$ under the same experimental settings were consistent with values reported in the literature, ${ }^{[24,28,29]}$ validating the method and providing a reference for the analysis of the impact of structure modifications in the stability of the ligand-lectin complex.

The ELLA data for homogeneous series of $\alpha$-Glc homoconjugates against ConA reflected the expected trend dictated by the known lectin selectivity and the classical multivalent effect. Thus, the monovalent $\alpha$-Glc derivatives 2 and 4 show $I_{50}$ values that are close to that of methyl $\alpha-D$ glucopyranoside $(11000 \pm 100 \mu \mathrm{M})$, with relatively short differences that are ascribable to aglycon effects. The $\mathrm{IC}_{50}$ value for the $\beta C D$ conjugate $4(9050 \mathrm{mM})$ has been taken as a reference for determination of relative potencies (RP) of binding to ConA and normalized relative potencies in $\alpha$-Glc molar basis (NRP), which allows a comparative evaluation of the multivalent effect . Thus, the hepta- and tetradeca-valent homologues 6 and 10 exhibit RP (NRP) values of 35 (5) and 323 (23), meaning that the $\alpha$-Glc glycotope is recognized with 5 -fold higher efficiency when going from valency 1 to valency 7 and that the efficiency further increases by 23 -fold when going to valency 14 (Table 1 )
Table 1. $I_{50}(\mu \mathrm{M})$, relative potency $(R P)$ and normalized relative potency (NRP) values for the inhibition of binding of HRP-ConA $A^{[a]}$ or HRP-PNA ${ }^{[b]}$ to a microtiter-anchored reference ligand by compounds 1-12 in competitive ELLA.

\begin{tabular}{|c|c|c|c|c|c|c|}
\hline & HRP-Con & & & HRP-PNA & & \\
\hline Comp. & $\begin{array}{l}\mathrm{IC}_{50} \\
(\mu \mathrm{M})\end{array}$ & $\begin{array}{l}\text { RP } \\
\text { (folds) }^{[c]}\end{array}$ & 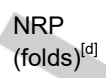 & $\begin{array}{l}1 \mathrm{C}_{50} \\
(\mu \mathrm{M})\end{array}$ & $\begin{array}{l}\text { RP } \\
\text { (folds) }^{[\mathrm{e}]}\end{array}$ & $\begin{array}{l}\text { NRP } \\
(\text { folds })^{[f]}\end{array}$ \\
\hline 1 & n.i. ${ }^{[g]}$ & n.i. & n.i. & $3100 \pm 50$ & 1.0 & 1.0 \\
\hline 2 & $7450 \pm 60$ & 1.2 & 1.2 & n.i. & n.i. & n.i. \\
\hline 3 & n.i. & n.i. & n.i. & n.i. & n.i. & n.i. \\
\hline 4 & $9050 \pm 90$ & 1.0 & 1.0 & n.i. & n.i. & n.i. \\
\hline 5 & n.i. & n.i. & n.i. & n.i. & n.i. & n.i. \\
\hline 6 & $260 \pm 25$ & 34.8 & 5.0 & n.i. & n.i. & n.i. \\
\hline 7 & $340 \pm 25$ & 26.6 & 7.6 & n.i. & n.i. & n.i. \\
\hline 8 & n.i. & n.i. & n.i. & n.i. & n.i. & n.i. \\
\hline 9 & n.i. & n.i. & n.i. & $10 \pm 2$ & 310 & 22.1 \\
\hline 10 & $28 \pm 2$ & 323 & 23.0 & n.i. & n.i. & n.i. \\
\hline 11 & $42 \pm 4$ & 215 & 30.7 & $19 \pm 2$ & 163 & 23.3 \\
\hline 12 & $88 \pm 2$ & 103 & 15.7 & n.i. & n.i. & n.i. \\
\hline
\end{tabular}

[a] Methyl $\alpha$-D-mannopyranoside and methyl $\alpha$-D-glucopyranosides afforded $I_{50}$ values of $1050 \pm 50$ and $11000 \pm 100 \mu \mathrm{M}$ in a parallel assay. [b] Lactose afforded an $\mathrm{IC}_{50}$ value of $1350 \pm 10 \mu \mathrm{M}$ in a parallel assay. [c] Referred to the $\beta C D$-linked monovalent control 3. [d] In $\alpha$-Glc molar basis. [e] Referred to the monovalent control 1. [f] In ONJ molar basis. [g] No inhibition detected at concentrations up to $5 \mathrm{mM}$.

The binding behaviour of homomultivalent ONJ $\mathrm{sp}^{2}$ iminosugar clusters towards PNA lectin can be similarly rationalized assuming a glycoligand-like behaviour. Indeed, although PNA is generally considered a galactose-binding lectin, glycopolymers and glycoclusters incorporating glucopyranosyl moieties with nitrogen functionalities at the primary position, structurally related to the ONJ motif, have been also shown to exhibit affinity towards PNA. ${ }^{[30]}$ Such recognition capabilities are only elicited after reaching a critical monomer density, which in the present case requires incorporation of the glycomimetic at both $\beta C D$ faces in 14-valent conjugates. Thus, whereas no PNA recognition could be evidenced for the primary-face ONJ clusters 5 and 8 even at relatively high $(5 \mathrm{mM})$ concentration, the corresponding $I_{50}$ value for $9(10 \pm 2 \mu \mathrm{M})$ represents a RP of 310 taken the monovalent derivative 1 as the reference, meaning a NRP of 22.1 in ONJ molar basis, a quite significant multivalent effect (Table 1).

Heterogeneity had a remarkable impact in ConA recognition, which is particularly evident when comparing the NRP values for $\alpha$-Glc homoclusters 6 and 10 ( 5 and 23) with the corresponding values for $\mathrm{ONJ} / \alpha-\mathrm{Glc}$ heteroclusters 7,11 and 12 (NRP 7.6, 30.7 and 15.7, respectively). The data are indicative 
of a $50 \%$ enhancement in the relative binding potency ( $\alpha-\mathrm{Glc}$ molar basis) when half of the $\alpha$-Glc glycotopes in $\mathbf{6}$ are replaced by ONJ moieties, a motif that is not a ligand for ConA. In the case of 12 and $\mathbf{1 1}$ the per-glucose affinity enhancements relative to 6 reach $310 \%$ and over $600 \%$, respectively. Regarding PNA, the combined display of ONJ and $\alpha$-Glc units on the primary face of the $\beta C D$ platform in $\mathbf{7}$ and $\mathbf{1 2}$ does not lead to functional ligands of this lectin, as also occurred for homocluster 5 . Heterocluster $\mathbf{1 1}$ keeps a binding affinity almost identical to that of the homogeneous ONJ homologue 9 when normalized to ONJ molar basis (NRP 23.3). Note, however, that this result means that efficient conjugate-lectin recognition was elicited with just half of the ONJ ligand density (Table 1). Control experiments discarded cross-selectivity between homogeneous $\alpha$-Glc glycoclusters $(\mathbf{6}, \mathbf{1 0})$ and PNA or homogeneous ONJ conjugates $(\mathbf{5}, \mathbf{8}, \mathbf{9})$ and ConA. The nonlinear behaviour observed for heterogeneous architectures must thus be interpreted in terms of synergistic cooperativity between binding and nonbinding motifs, which represents the first evidence of a glycotope/inhitope heterocluster effect.

\section{Impact of (hetero)multivalency in glycosidase inhibition}

Although the monovalent $\alpha$-glucopyranosides $\mathbf{2}$ and $\mathbf{4}$ are substrates of the $\alpha$-glucosidases maltase and isomaltase, control experiments showed that the $\alpha$-Glc moieties displayed at the periphery of $\beta C D$-centered homo $(6,10)$ or heteromultivalent derivatives $(\mathbf{7}, \mathbf{1 1}, \mathbf{1 2})$ were not susceptible to hydrolysis by any of these two enzymes (gas chromatography monitoring). All monovalent and (hetero)multivalent $\alpha$-Glc conjugates remained also unmodified after incubation with $\alpha$-mannosidase, as expected from the mismatching configurational relationship. Alterations in substrate hydrolysis kinetics in their presence must then be ascribed to interactions with the enzyme but not to substrate-like behavior. The inhibition constants $\left(K_{\mathrm{i}}\right)$ values of the whole compound library 1-12 against maltase, isomaltase and $\alpha$-mannosidase are collected in Table 2.

Table 2. $K_{\mathrm{i}}$ values $[\mu \mathrm{M}]$ of the mono (1-4) and (hetero)multivalent $\beta C D$ glyco(mimetic)clusters (5-12). ${ }^{[a]}$

\begin{tabular}{llll}
\hline \multicolumn{4}{l}{$K_{\mathrm{i}}$ values $(\mu \mathrm{M})^{[\mathrm{b}]}$} \\
\hline $\mathbf{1}$ Compound & Maltase & Isomaltase & $\alpha$-Mannosidase \\
\hline $\mathbf{2}$ & $2.6 \pm 0.1$ & $5.1 \pm 0.2$ & $596 \pm 10$ \\
$\mathbf{3}$ & n.d. $^{[\mathrm{c}]}$ & n.d. & n.i. $^{[\mathrm{d}]}$ \\
$\mathbf{4}$ & $53 \pm 3$ & $61 \pm 3$ & n.i. \\
$\mathbf{5}$ & n.d. & n.d. & n.i. \\
$\mathbf{6}$ & $2.9 \pm 0.1$ & $1.9 \pm 0.1$ & $23.0 \pm 0.5$ \\
& n.i. & n.i. & $590 \pm 12$ \\
& $1.4 \pm 0.1$ & $1.4 \pm 0.1$ & $506 \pm 10$
\end{tabular}

\begin{tabular}{llll}
8 & $40 \pm 3$ & $65 \pm 5$ & $35 \pm 2$ \\
9 & $1.7 \pm 0.1$ & $2.0 \pm 0.1$ & $2.5 \pm 0.2$ \\
10 & $524 \pm 15$ & $326 \pm 10$ & $351 \pm 10$ \\
11 & $4.2 \pm 0.3$ & $5.6 \pm 0.3$ & $70 \pm 6$ \\
12 & $0.82 \pm 0.05$ & $1.6 \pm 0.1$ & $5.1 \pm 0.3$ \\
\hline
\end{tabular}

[a] Inhibition was competitive in all cases. [b] Results in triplicate were used for determination of $K_{\mathrm{i}}$ values; the corresponding standard deviations are indicated. [c] Not determined. [d] No inhibition was observed at $1 \mathrm{mM}$ concentration $(\mathrm{Ki}>1000 \mu \mathrm{M})$.

We have previously provided evidence supporting that most of the binding energy of mono and multivalent inhibitors to $\alpha$-mannosidase arise from interactions involving the same region in the protein, namely the aglycon $(-1)$ site in the catalytic pocket. $^{[17,25]}$ This situation is analogous to that generally encountered in carbohydrate-lectin recognition events. Consequently, the relative inhibition potency (RIP) enhancement on moving from the monovalent reference (1) to (hetero)multivalent analogues (determined as the quotient between the corresponding $K_{\mathrm{i}}$ values in Table 2), normalized on per-ONJ molar basis (NRIP), is a good indication of the inhibitory multivalent effect intensity (Table 3). On the contrary, in the case of maltase or isomaltase monovalent and (hetero)multivalent inhibitors are expected to target different sites in the protein: monovalent inhibitors locate at the aglycone site whereas multivalent partners probably block access to the catalytic site by attaching at non-glycone regions. ${ }^{[17,25]}$ This scenario prevents a similar approach for comparative evaluation of potential (hetero)multivalent effects. Control experiments indicated that homocluster $\mathbf{8}$, with a 3.5 nominal valency, is the lowest-valency ONJ derivative in the series exhibiting the nonglycone binding mode. For normalization purposes, we have considered that the $K_{\mathrm{i}}$ values measured for 8 against maltase and isomaltase (40 and $65 \mu \mathrm{M}$, respectively; Table 2) correspond, formally, to 3.5 -folds the inhibitory potency of a single ONJ unit interacting at the same enzyme region. Note that this would imply a mere statistic effect for $\mathbf{8}$. Although we are aware that this assumption underestimates multivalent effects when considering absolute RIP/NRIP values, it provides a good basis for comparison. The corresponding calculated RIP and NRIP values for the ensemble of hetero(multivalent) conjugates are collected in Table 3.

Table 3. Relative inhibition potency (RIP; folds) and normalized relative inhibition potency (NRIP; folds) values for the inhibition of yeast maltase and isomaltase and Jack bean $\alpha$-mannosidase by (hetero)multivalent $\beta C D$ glyco(mimetic)clusters 5-12.

\begin{tabular}{|c|c|c|c|c|c|c|}
\hline \multirow[t]{2}{*}{ Compound } & \multicolumn{2}{|c|}{ Maltase } & \multicolumn{2}{|c|}{ Isomaltase } & \multicolumn{2}{|c|}{$\alpha$-Mannosidase } \\
\hline & $\mathrm{RIP}^{[\mathrm{a}]}$ & NRIP ${ }^{[b]}$ & $\mathrm{RIP}^{[\mathrm{c}]}$ & $N R I P[b]$ & $\operatorname{RIP}^{[\mathrm{d}]}$ & $N R I P^{[b]}$ \\
\hline
\end{tabular}




\begin{tabular}{lllllll}
$\mathbf{5}$ & 48.2 & 6.9 & 119 & 17 & 25.9 & 3.7 \\
$\mathbf{6}$ & n.i. ${ }^{[\text {[e] }}$ & n.a. ${ }^{[\text {] }}$ & n.i. & n.a. & 1 & n.a. \\
$\mathbf{7}$ & 100 & 28.6 & 162 & 46.3 & 1.2 & 0.34 \\
$\mathbf{8}$ & 3.5 & 1 & 3.5 & 1 & 17 & 4.8 \\
$\mathbf{9}$ & 82 & 5.9 & 113 & 8.1 & 238 & 17 \\
$\mathbf{1 0}$ & 0.27 & n.a. & 0.7 & n.a. & 1.7 & n.a. \\
$\mathbf{1 1}$ & 33.3 & 4.8 & 40.5 & 5.8 & 8.5 & 1.2 \\
$\mathbf{1 2}$ & 171 & 24.4 & 142 & 20.3 & 117 & 16.7 \\
\hline
\end{tabular}

[a] Referred to the formal contribution of each ONJ motif in 8 to the inhibition potency against maltase $\left(K_{\mathrm{i}} 140 \mu \mathrm{M}\right)$. [b] In ONJ molar basis. [c] Referred to the formal contribution of each ONJ motif in 8 to the inhibition potency against isomaltase $\left(K_{\mathrm{i}} 227 \mu \mathrm{M}\right)$. [d] Referred to the monovalent reference control $1\left(K_{\mathrm{i}} 596 \mu \mathrm{M}\right)$. [e] No inhibition was observed at $1 \mathrm{mM}$ concentration $\left(K_{\mathrm{i}}>1000 \mu \mathrm{M}\right)$. [f] Do not apply.

Maltase and isomaltase exhibited analogous inhibition susceptibility trends when profiled against 1-12. The monovalent ONJ control 1 is a potent inhibitor of both enzymes $\left(K_{\mathrm{i}} 2.6\right.$ and $5.1 \mu \mathrm{M}$, respectively). The inhibitory potency decreases by above 10 -fold when the inhitope is connected to the $\beta C D$ scafold (3; $K_{\mathrm{i}} 53$ and $61 \mu \mathrm{M}$ ), probably due to unfavourable steric contacts between the cyclooligosaccharide and the proteins upon hosting the glycomimetic at the active site. In apparent contradiction, the much bigger multiconjugates $5,7,9,11$ and 12 behaved as low $\mu \mathrm{M}$-to-nM inhibitors. Homovalent $\alpha$-Glccoated glycoclusters were either no (6) or very weak (10) inhibitors (Table 2). The optimal per-ONJ efficiency among homoconjugates is reached for the heptavalent derivative 5 , with NRIP values of 6.9 and 17 for maltase and isomaltase, respectively. Remarkably, the simultaneous presentation of ONJ (inhibitory) and $\alpha$-Glc (non-inhibitory) motifs in 7 further boosted the inhibitory potency, with NRIP values scaling up to 28.6 and 46.3. In other words, each ONJ inhitope in heterocluster 7 is recognized 4-fold (for maltase) or 2.7-fold (for isomaltase) more effectively that in homovalent cluster $\mathbf{5}$, with an overall analogous architecture, which represents the first evidence of a heteromultivalent inhibitory effect. A similar synergistic action is observed in the case of compound 12 (NRIP 24.4 and 20.3 against maltase and isomaltase): both homocluster 5 and heterocluster 12 expose seven ONJ moieties at the primary $\beta C D$ rim, but the simultaneous presence of seven $\alpha$-Glc moieties in 12 translates into significantly higher inhibitory potencies (Table 3).

The monovalent control 1 was instead a very poor inhibitor of Jack bean $\alpha$-mannosidase $\left(K_{i} 596 \mu \mathrm{M}\right)$ and the corresponding $\beta C D$ conjugate 3 showed no measurable inhibition at $1 \mathrm{mM}$ concentration. In sharp contrast, the hepta- and tetradecavalent glycomimetic homoclusters $\mathbf{5}$ and $\mathbf{9}$ behaved as strong inhibitors of the enzyme, with $K_{\mathrm{i}}$ values of 23 and $2.5 \mu \mathrm{M}$, respectively (Table 2). The bouquet-type tetradecavalent ONJ homocluster 9 showed the highest multivalent inhibitory effect, with a RIP of

238, corresponding to a NRIP (ONJ molar basis) of 17 . To our surprise, the combined presentation of ONJ and $\alpha$-Glc substituents in $\mathbf{1 1}$ was strongly detrimental for $\alpha$-mannosidase inhibition (NRIP 1.2-fold). An even more dramatic antagonistic effect was observed when comparing the primary rim-substituted homoconjugates 5 or $\mathbf{8}$ (NRIP 3.84 .8 ) with heteroconjugate 7 (NRIP 0.34). Interestingly, compound 12, with the same total and partial valencies than 11, turned out to be a much potent inhibitor of $\alpha$-mannosidase $\left(K_{\mathrm{i}} 5.1 \mu \mathrm{M}\right)$, meaning a NRIP of 16.7 , about five-fold higher as compared with the homo-heptavalent derivative 5 (Table 3), suggesting that architectural parameters can significantly impact multivalent and heteromultivalent inhibition of $\alpha$-mannosidase.

The ensemble of lectin binding and glycosidase inhibition data clearly illustrates that (hetero)mutlivalency affects simultaneously and in different manners both types of events. Although the inhibition potency enhancements observed for homomultivalent ONJ conjugates suggest that similar mechanisms may operate in multivalent enzyme inhibition and in glycocluster-lectin binding, the much higher sensitiveness of the enzymes to heteromultivalency points to significant differences in the binding modes that need to be investigated. To get information on this aspect, different cross-linking assays were next conducted.

\section{Impact of (hetero)multivalency in lectin cross-linking abilities: Preferred lectin binding modes}

Although both ConA and PNA are tetramers at the closeto-neutral $\mathrm{pH}$ used in ELLA, ${ }^{[31,32]}$ the presence of the voluminous HRP-tag disable lectin cross-linking and chelate-type binding modes. Essentially, the $\mathrm{IC}_{50}$ data in Table 1 originate from interactions of the glyco(mimetic) ligands with a single binding site in the lectin. ${ }^{[3]]}$ The relative cross-linking potential of the homo and heterovalent conjugates was assessed by two-site (sandwich-type) ELLA experiments. In this case, the ability of the glycoligands to bridge the HRP-labelled lectin in the solution and unlabelled (therefore cross-linkable) lectin fixed in the plate is determined. ${ }^{[34]}$ As expected, the 14-valent $\alpha$-Glc glycocluster 10 exhibited the highest ConA cross-linking potential, which was set at $100 \%$ for a $500 \mu \mathrm{M}$ concentration. The rest of homo- or heterovalent $\alpha$-Glc-displaying conjugates exhibited relative cross-linking abilities that followed their lectin binding efficacy, i.e. $10>11>12>6>7$ (Figure $3 \mathrm{~A}$ ). A similar two-site ELLA test using PNA and HRP-PNA let confirm the cross-linking potential of the two-face ONJ-displaying clusters 9 and 11 (Figure $3 \mathrm{~B}$ ).

A

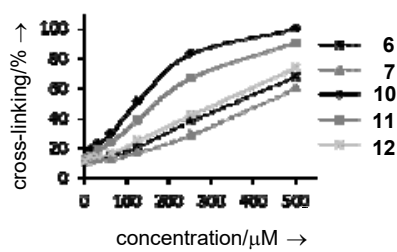

B

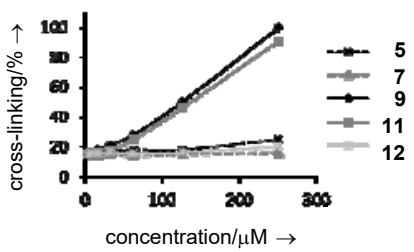


Figure 3. Relative ConA (A) and PNA (B) cross-linking capability plots of $\alpha$ Glc/ONJ homo and heteromultivalent conjugates determined by two-site ELLA

Analogous ConA cross-linking experiments conducted in the presence of excess of mono or homomultivalent ONJ conjugates $(\mathbf{1}, \mathbf{3}, \mathbf{5}, \mathbf{9} ; 500 \mu \mathrm{M})$ led to virtually superimposable plots to those shown in Figure $3 \mathrm{~A}$. Reciprocally, the presence of mono or homomultivalent $\alpha$-Glc conjugates $(2,4,6,10 ; 500 \mu \mathrm{M})$ did not alter the PNA cross-linking plots for the homovalent ONJ derivative 9 or the heterovalent analogue 11 shown in Figure $3 \mathrm{~B}$ These results discard the presence of secondary sites for ONJ in ConA or for $\alpha$-Glc in PNA that could promote a "carbohydrate module effect" mechanism, as proposed for heteroglycopolymers targeting different subsites in oligosaccharide binding lectins (Figure $4 \mathrm{~A}$ ). ${ }^{[35]}$ Two alternative mechanistic hypotheses have been advanced to account for the lectin affinity enhancement promoted for nonbinding sugar epitopes in heteromultivalent systems, namely entropicallydriven stabilisation of lectin-heterocluster complexes through assisted sliding of the putative glycotopes over the binding site ${ }^{[8]}$ (Figure $4 \mathrm{~B}$ ) or induction of a favourable orientation of the binding motifs resulting in steric shielding and an overall increased in affinity ${ }^{[11]}$ (Figure $4 \mathrm{C}$ ). The latter scenario is expected to penalize significantly the lectin bridging capabilities of the heteroconjugates as compared to homoconjugates. Although the two-site ELLA plots (Figure 3) revealed a moderate depletion of the lectin cross-linking efficiency for heteroclusters, compounds 7, 11 and 12 still keep substantial ConA crosslinking potential, as well as 11 against PNA, suggesting that both mechanisms, assisted sliding and steric shielding, may operate simultaneously in these systems.
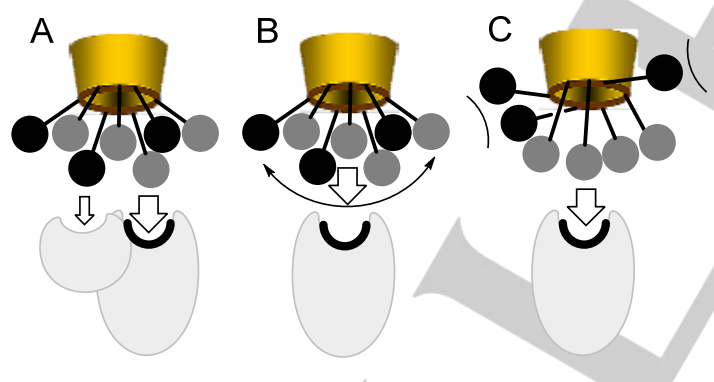

Figure 4. Schematic representation of the three possible mechanisms advanced to account for the heterocluster effect: $(A)$ the presence of high and low affinity binding sites in the lectin for different carbohydrate modules (carbohydrate module effect), (B) entropic stabilization of the heteromultivalent ligand-lectin complex by fast sliding processes, and (C) conformational preorganization of the binding motif and steric shielding leading to higher binding affinity. Grey and black balls represent ONJ and $\alpha$-Glc substituents, respectively. The primary binding site in the lectin is highlighted in black. The experimental data support that the two later mechanisms may simultaneously act for the $\beta C D$-centred heteromultivalent clusters in this study.

\section{Competitive lectin-glycosidase binding assays: Mapping (hetero)multivalent glycosidase binding modes}

The capability of several of the $\beta C D$-scaffolded glyco(mimetic)clusters evaluated in this work of simultaneously cross-linking lectins and inhibiting glycosidases enables a competitive assay in which the efficiency of the tested enzyme to sequester the ligand, therefore preventing complementary lectin clustering, is monitored in the absence and in the presence of inhibitors of the enzyme. ${ }^{[17,25]}$ By using inhibitors targeting the glycone and/or aglycone sites in the glycosidase, the involvement of these regions in binding to the (hetero)multivalent conjugate can be mapped (Figure 5).

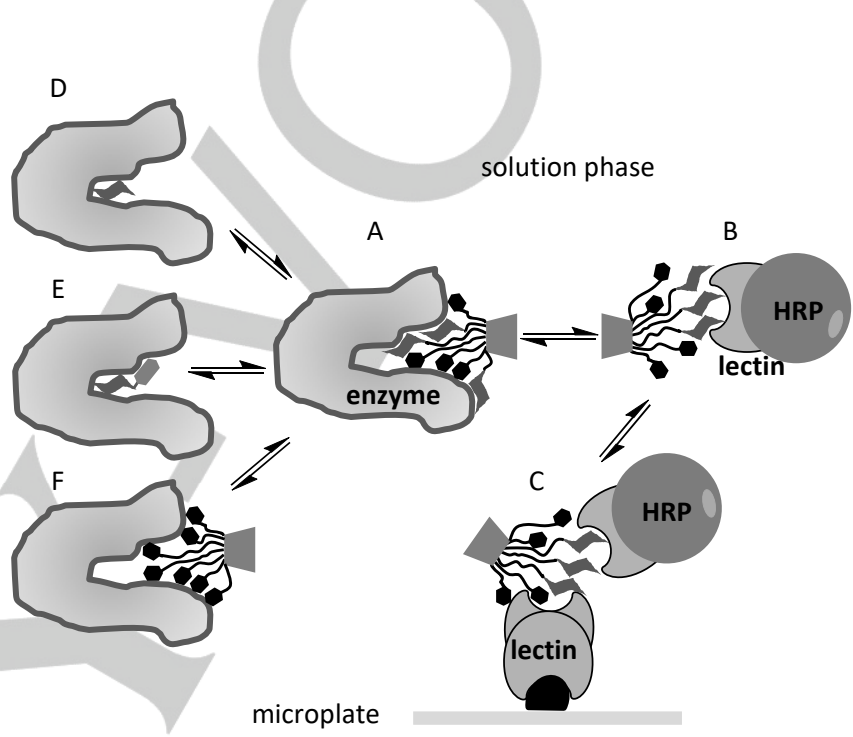

Figure 5. Schematic representation of the enzyme-lectin competitive assay configuration. In the absence of a third species, an equilibrium between the enzyme/ligand complex (A) and the lectin-HRP/ligand (B) is established in the solution phase; as the concentration of the enzyme increases, the available ligand to cross-link the lectin anchored in the microplate decreases, which can be evaluated in a two-site ELLA. In the presence of an enzyme inhibitor, formation of the corresponding enzyme-inhibitor complex (D-F) will compete with formation of complex $A$, and the lectin cross-linking potential will be enhanced. By employing inhibitors occupying the glycone site (D), simultaneously the glycone and aglycone sites (E) or a wider enzyme surface $(F)$, the biding mode of the tested ligand can be probed.

In the case of the ONJ homocluster $\mathbf{9}$, its potential to cross-link PNA (in the microplate) and HRP-PNA (in the solution) at $125 \mu \mathrm{M}$ concentration, as determined in the two-site ELLA experiment (Figure $3 \mathrm{~B}$; set at $100 \%$ ), experienced a hyperbolic decrease with increasing concentrations of maltase or $\alpha$-mannosidase. This is in agreement with the existence of a reversible exchange of the multivalent compound between the two protein species in the solution phase. An identical experiment carried out for maltase in the presence of three different concentrations of the $\mathrm{sp}^{2}$-iminosugar-type glycomimetic $13{ }^{[36]}$ a glycone-type inhibitor of this enzyme, showed a very minor alteration of the above equilibrium (Figure $6 \mathrm{~A}$ ), strongly supporting that the glycone $(-1)$ catalytic site is only marginally participating, if any, in the stabilisation of the 9:maltase complex. In sharp contrast, the pseudodisaccharide inhibitor $14,{ }^{[37]}$ which simultaneously occupies the glycone $(-1)$ and aglycone $(+1)$ 
sites of maltase, or the multivalent inhibitor $\mathbf{5}$ (which does not cross-link PNA but binds to maltase) almost completely restored the lectin clustering capabilities (Figure $6 \mathrm{~B}$ and $\mathrm{C}$ ). Analogous $\mathrm{PNA} / \alpha$-mannosidase competitive experiments were conducted in the absence and presence of glycone (15), ${ }^{[38]}$ glycone/aglycone $(16)^{[17]}$ and multivalent (5) inhibitors of $\alpha$ mannosidase that are not ligands of the lectin (Figure $6 \mathrm{D}-\mathrm{F}$ ). The glycone-type inhibitor 15 was already able to very significantly re-establish the PNA cross-linking capabilities of $\mathbf{9}$ to a very significant extent (Figure $6 \mathrm{D}$ ), highlighting its decisive implication in multivalent $\alpha$-mannosidase inhibition.
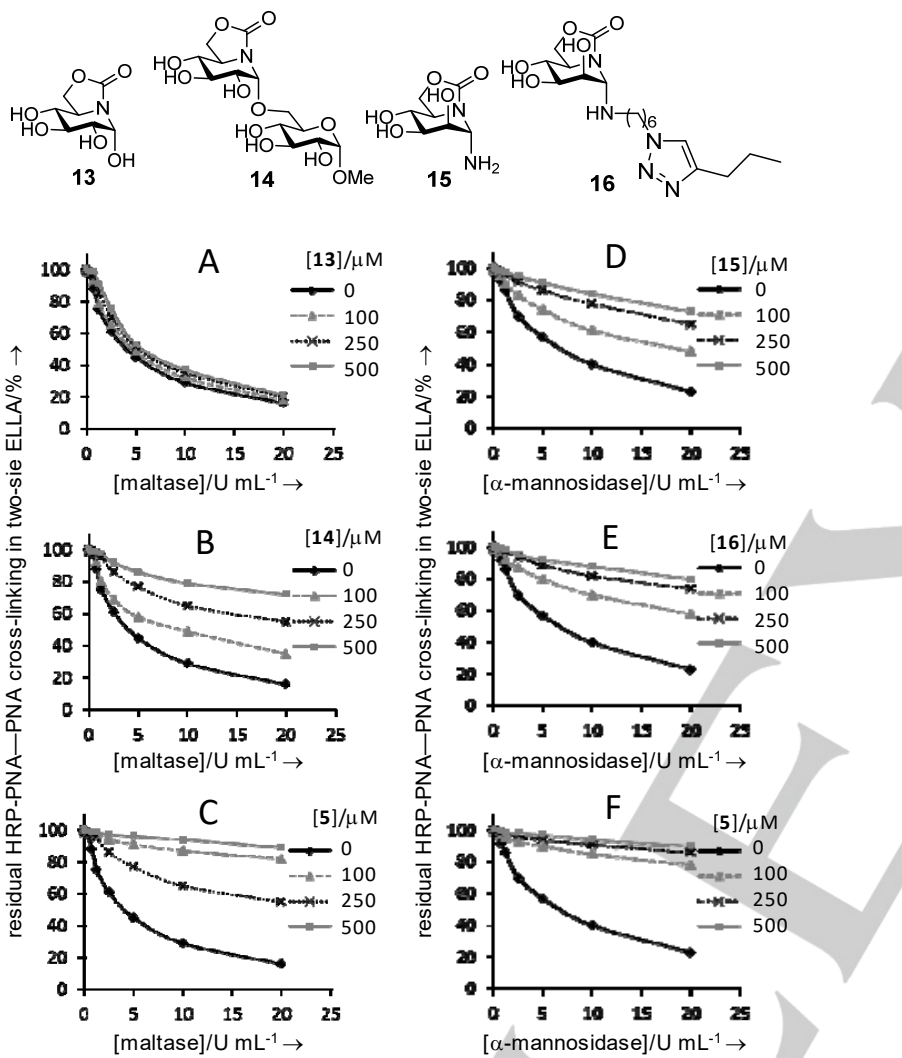

Figure 6. Plots of the inhibition of the PNA-HRP-PNA cross-linking mediated by the ONJ $\beta C D$ homocluster 9 in two-site ELLA tests by increasing amounts of maltase (A-C) or $\alpha$-mannosidase (D-F). Data were collected in the absence and presence of 100,250 or $500 \mu \mathrm{M}$ concentrations of the aglycon-type inhibitors 13 or 15 (A and D), the glycone/aglycone-type inhibitors 14 or 16 (B and $E$ ), or the multivalent inhibitors 5 ( $C$ and $F$ ). Each point represents the mean of three independent determinations (S.D. 12-18\%).

Identical assays were completed for the rest of (hetero)multivalent $\beta C D$ conjugates exhibiting dual $\mathrm{PNA} /$ maltase (11), PNA/ $\alpha$-mannosidase $(11)$, ConA/maltase $(7,11,12)$ or ConA/ $\alpha$-mannosidase (12) strong recognition abilities. The $\alpha$-Glc homoglycocluster 10, although a rather modest inhibitor of $\alpha$ mannosidase $\left(K_{\mathrm{i}} 351 \pm 10 \mu \mathrm{M}\right)$, was also evaluated in the ConA/ $\alpha$-mannosidase competitive assay for comparative purposes. The capacities of increasing concentrations of the reference glycone (13 or 15$)$, glycone/aglycone (14 or 16$)$ or multivalent (5) inhibitors of maltase or $\alpha$-mannosidase to interfere in the formation of the corresponding glyco(mimetic)cluster-enzyme complex, as determined by the residual lectin cross-linking capabilities at the maximum enzyme concentration tested $\left(20 \mathrm{U} \mathrm{mL}^{-1}\right)$, are represented in Figure 7.

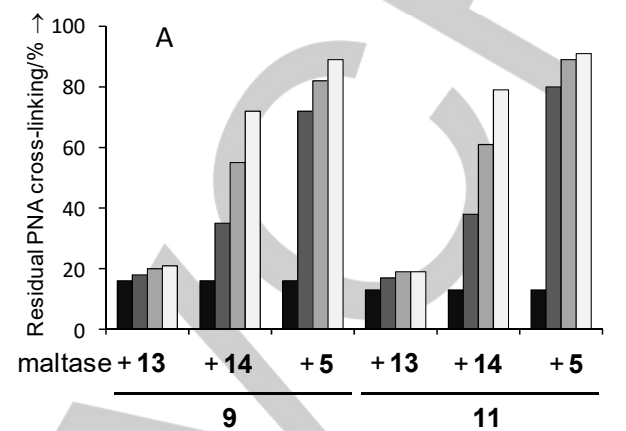

[Inhibitor $] / \mu \mathrm{M}$

-

100

$\square 250$

$\square 500$

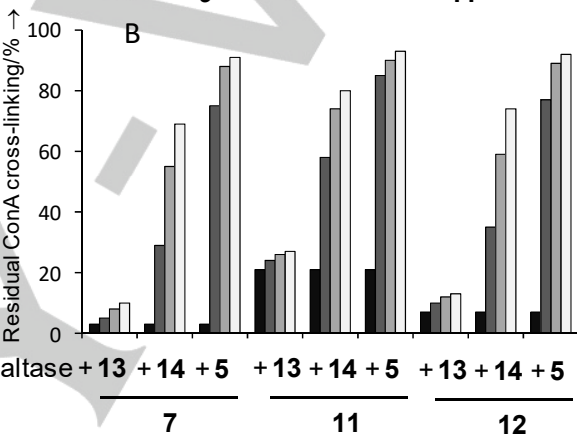

[Inhibitor $] / \mu \mathrm{M}$

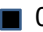

100

$\square 250$

$\square 500$

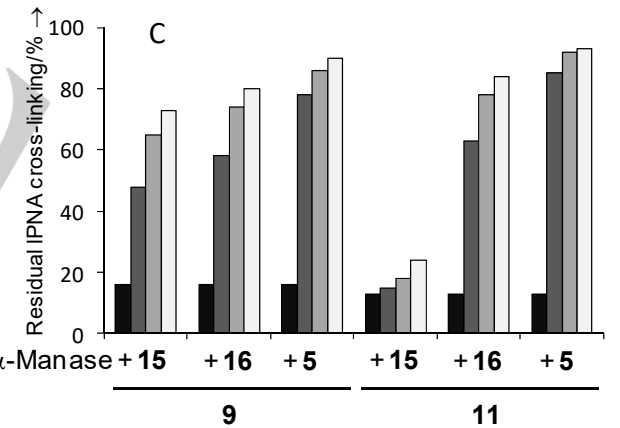

[Inhibitor] $/ \mu \mathrm{M}$

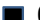

- 100

$\square 250$

$\square 500$

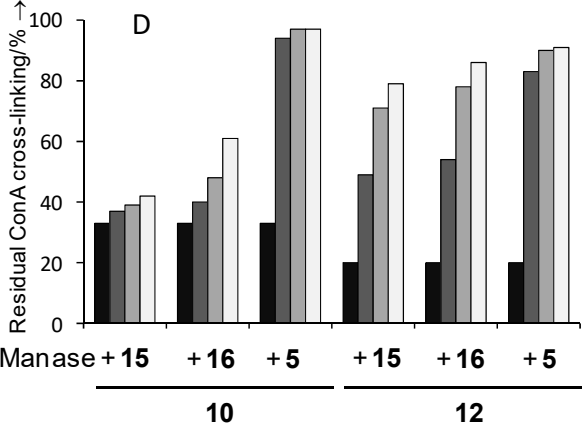

[Inhibitor] $/ \mu \mathrm{M}$

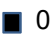

- 100

$\square 250$

$\square 500$

Figure 7. Residual PNA-HRP-PNA (A and C) or ConA-HRP-ConA crosslinking $(B$ and $D)$ mediated by the indicated glyco(mimetic)clusters $(125 \mu \mathrm{M})$ in the presence of maltase $(A$ and $B$ ) or $\alpha$-mannosidase ( $\alpha$-Manase; $C$ and $D$ ) at $20 \mathrm{U} \mathrm{mL}^{-1}$. Determinations have been conducted in the absence and in the presence of increasing amounts of aglycone- (13 or 15), glycone/aglycone (14 
or 16) and multivalent-type (5) inhibitors of the enzymes. Each bar represents the mean of three independent determinations (S.D. $15-20 \%$ )

The ensemble of data strengthens the idea that monovalent and (hetero)multivalent inhibitors target different sites in maltase. Thus, no competition between the glycone-type inhibitor 13 and multiconjugates 7, 9, 11 or 12 was observed (see Figure $7 \mathrm{~A}$ and $\mathrm{B}$ ). Binding of the same (hetero)multivalent derivatives to maltase was instead very sensitive to the presence of the glycone/aglycone inhibitor $\mathbf{1 4}$ or the multivalent inhibitor 5. A binding mode involving the aglycone site in priority (for which the affinity follows the trend $\mathrm{ONJ}>>\alpha-\mathrm{Glc}$ ), reinforced by additional interactions with other nonglycone subsites (with the opposite affinity trend) can account for these observations (Figure $8 \mathrm{~A}$ ). This mechanism agrees with the fact that the $\alpha$-Glc homoclusters $\mathbf{6}$ and $\mathbf{1 0}$ are no or very weak inhibitors of maltase and isomaltase, but can however significantly enhance the inihibitory potency when present in heterodisplays with ONJ motifs (Tables 2 and 3). The preferred implication of the aglycone site is also consistent with the competitive character of the inhibition.

In the case of Jack bean $\alpha$-mannosidase two different scenarios were apparent. In agreement with previous observations, the glycone site was found to be critically involved in binding of ONJ homomultivalent inhibitors, since the glyconetype inhibitor $\mathbf{1 5}$ was already able to efficiently restore the lectin cross-linking capabilities of $\mathbf{9}$ in the corresponding $P N A / \alpha-$ mannosidase competitive assay (Figure $7 \mathrm{C}$ ). A similar situation was encountered for the heterovalent derivative 12 (Figure $7 \mathrm{D}$ ). Nonglycone subsites probably provide additional secondary interactions (Figure $8 \mathrm{~B}$ ). In sharp contrast, the reference glycone-type inhibitor 15 did not affect binding of the ONJ/ $\alpha-G l c$ statistic heteroclusters 7 (Figure $7 \mathrm{~B}$ ) and 11 (Figure $7 \mathrm{C}$ ) or the $\alpha$-Glc homogeneous glycocluster 10 (Figure 7 D) to $\alpha$ mannosidase. In those cases, enzyme binding was very sensitive to the presence of the glycone/aglycone inhibitor $\mathbf{1 6}$ or the multivalent inhibitor $\mathbf{5}$. These observations strongly suggest that the presence of the $\alpha$-Glc motif in 7, 10 and 11 , but not in 12 , elicits a shift from the binding mode entailing the glycone site to another one in which this site is no longer available. The negative heterovalent effect observed when comparing the NIRP values of $\mathbf{5}$ or $\mathbf{9}$ with $\mathbf{7}$ or $\mathbf{1 1}$ (Table 3) reflects the much lower efficiency of the second binding mode.

In principle, the glycone site of a glycosidase is much more demanding than the aglycone or other nonglycone subsites in terms of the structural and architectural requirements that a complementary binding partner must fulfil. For instance, it is known that the presence of a single $\mathrm{N}$-acetylglucosamine substituent in high mannose oligosaccharide substrates can totally abolish processing by Jack bean $\alpha$-mannosidase by impeding the proper orientation of the terminal $\alpha$-manopyranosyl units. ${ }^{[39]}$ In the $\beta C D$ heteroconjugates 7 and 11 the presence of the $\alpha$-Glc units in different arms seem to exert a similar action, thwarting proper accommodation of the ONJ inhitope in the glycone site and permitting only interactions with aglycone/nonglycone subsites, which in practice turns off efficient enzyme inhibition (Figure $8 \mathrm{C}$ ). In case of 12, the higher flexibility of the dendritic architecture allows conformations in which the ONJ and $\alpha$-Glc moieties are accommodated in opposite space regions. In the most favourable situation, a symmetrical segregated distribution can be achieved, which might benefit from a heptavalent presentation of the ONJ inhitopes (like in 5) capable of multivalently interacting with the glycone site of $\alpha$-mannosidase and probably benefitting from additional interactions of the $\mathrm{ONJ} / \alpha-\mathrm{Glc}$ motifs with other nonglycone subsites (Figure $8 \mathrm{D}$ ).
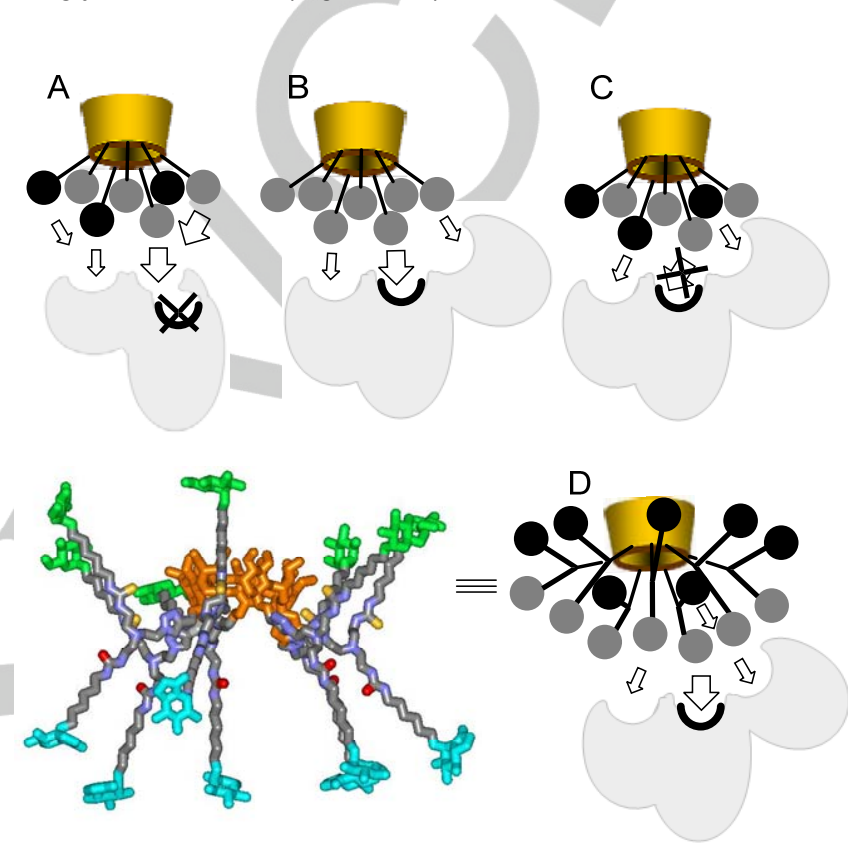

Figure 8. Schematic representation of the mechanisms proposed to account for the inhibitory heterocluster effect. Grey and black balls represent ONJ and $\alpha$-Glc substituents, respectively. (A) In maltase or isomaltase, the glycone site (highlighted in black) is not accessible and interactions occur at the aglycone (preferentially involving ONJ moieties) and other nonglycone subsites (preferentially involving $\alpha$-Glc units). (B) The implication of the glycone site is critical in the inhibition of $\alpha$-mannosidase by ONJ homoclusters. (C) The presence of $\alpha$-Glc substituents in heteroclusters prevents accommodation of ONJ units in the glycone site. (D) The dendritic architecture of heterocluster 12 is compatible with conformations allowing favourable ONJ-glycone site interactions, resulting in enhanced $\alpha$-mannosidase inhibition. The 3D molecular model of $\mathbf{1 2}$ in the fully extended, segregated conformation is shown (ONJ units in light blue; $\alpha$-Glc units in light green; $\beta C D$ scaffold in orange).

\section{Conclusions}

Taken together, the ensemble of results here discussed substantiates the vision that (hetero)multivalency promotes binding modes that share significant analogies in both lectins and glycosidases. Beyond this notion, the work here presented demonstrates that (hetero)multivalent displays can simultaneously act on lectins and glycosidases in a multimodal manner and that a given glycotope or inhitope moiety may elicit different responses depending on the presence or not of a second, a priori innocent, glyco(mimetic) motif. By changing total and partial valencies and adjusting the overall topology of the 
(hetero)multivalent construct, "on" or "off" states for a range of lectins and glycosidases can be activated, markedly altering the selectivity pattern encountered for monovalent derivatives. It is important to emphasize that although invoking a lectin-like behaviour to justify the responses of glycosidases to (hetero)multivalency is appealing, identifying the operating mechanisms is essential for a rigorous understanding. In this sense, the work here presented provides unique experimental tools for the combined analysis of (hetero)multivalent effects in lectin binding and glycosidase inhibition. Most importantly, it delivers a rational for the disturbing observation that artificial (hetero)multivalent carbohydrate displays, profusely investigated to interfere in lectin-mediated biological events, can eventually exhibit a priori unforeseen glycosidase inhibitory properties. Further work in this sense should ascertain the repercussions of the multimodal ligand character of (hetero)multivalent glyco(mimetic)clusters in biological systems.

\section{Experimental Section}

For the detailed description of the synthetic procedures, the analytical and spectroscopic data and the biochemical assays, see the Supporting Information.

\section{Acknowledgements}

This study was supported by the Spanish Ministerio de Economía y Competitividad (contract numbers SAF2016-76083$\mathrm{R}$ and CTQ2015-64425-C2-1-R), the Junta de Andalucía (contract number FQM2012-1467) and the European Regional Development Funds (FEDER and FSE). Technical assistance from the research support services of the University of Sevilla (CITIUS) is also acknowledged.

Keywords: carbohydrates $\bullet$ glycomimetics $\bullet$ heteromultivalency - inhibitors $\cdot \mathrm{sp}^{2}$-iminosugars

[1] a) A. F. F. R. Nardy, L. Freire-de-Lima , C. G. Freire-de-Lima, A. Morrot Front. Oncol. 2016, 6, 54; b) K. Ohtsubo, J. D. Marth, Cell 2006, 126, 855-867; c) B. E. Collins, J. C. Paulson, Curr. Opin. Chem. Biol. 2004 8, 617-625; d) P. Albersheim, B. S. Valent, J. Cell. Biol. 1978, 78, 627643

[2] a) H.-J. Gabius, J. C. Manning. J. Kopitz, S. André, H. Kaltner, Cell. Mol. Life Sci. 2016, 73, 1989-2016; b) D. Solís, N. V. Bovin, A. P. Davis, J. Jiménez-Barbero, A. Romero, R. Roy, K. Smetana Jr., H.-J. Gabius, Biochim. Biphys. Acta 2015, 1850, 186-235; c) H.-J. Gabius, H. C Siebert, S. André, J. Jiménez-Barbero, H. Rüdiger, ChemBioChem, 2004, 5, 740-764.

[3] a) G. J. Davies, A. Planas, C. Rovira, Acc. Chem. Res. 2012, 45, 308316; b) G. Speciale, A. J Thompson, G. J. Davies, S. J. Williams, Curr. Opin. Struct. Biol. 2014, 28, 1-13.

[4] a) M. Mammen, S.-K. Choi, G. M. Whitesides, Angew. Chem. Int. Ed. 1998, 37, 2754-2794; b) C. R. Bertozzi, L. L. Kiessling, Science 2001 291, 2357-2364; c) J. J. Lundquist, E. J. Toone, Chem. Rev. 2002, 102, 555-578; d) L. L. Kiessling, J. E. Gestwicki and L. E. Strong, Angew. Chem., Int. Ed. 2006, 45, 2348; e) C. Fasting, C. A. Schalley, M. Weber,
O. Seitz, S. Hecht, B. Koksch, J. Dernedde, C. Graf, E.-W. Knapp, R. Haag, Angew. Chem., Int. Ed. 2012, 51, 10472-10498.

[5] For selected recent reviews on multivalent glycoarchitectures as poten lectin ligands, see: a) N. Spinelli, E. Defrancq, F. Morvan, Chem. Soc. Rev. 2013, 42, 4557-4573; b) K. Hatano, K. Matsuoka, D. Terunuma, Chem. Soc. Rev. 2013, 42, 4574-4598; c) M. C. Galan, P. Dumy, O Renaudet, Chem. Soc. Rev. 2013, 42, 4599-4612; d) F. Sansone, A. Casnati, Chem. Soc. Rev. 2013, 42, 4623-4639; e) Y. M. Chabre, R. Roy, Chem. Soc. Rev. 2013, 42, 4657-4708; f) J.-L. Reymond, M. Bergmann, T. Darbre, Chem. Soc. Rev. 2013, 42, 4814-4822.

[6] A few monovalent glycomimetics with high affinity towards complementary lectins have also been developed; for a review, see: S Cecioni, A. Imberty, S. Vidal, Chem. Rev. 2015, 115, 525-561.

[7] a) R. Lahiri, A. A. Ansari, Y. D. Vankar, Chem. Soc. Rev. 2013, 42 5102-5118; b) T. M. Gloster, D. J. Vocadlo, Nat. Chem. Biol. 2012, 8 683-694; c) T. M. Wrodnigg, A. E. Stütz, Adv. Carbohydr. Chem. Biochem. 2011, 66, 187-298.

[8] M. Gómez-García, J. M. Benito, D. Rodríguez-Lucena, J. Yu, K. Chmurski, C. Ortiz Mellet, R. Gutiérrez Gallego, A. Maestre, J. Defaye, J. M. García Fernández, J. Am. Chem. Soc. 2005, 127, 7970-7971.

[9] M. Gómez-García, J. M. Benito, R. Gutiérrez-Gallego, A. Maestre, C. Ortiz Mellet, J. M. García Fernández, J. L. Jiménez Blanco, Org. Biomol. Chem. 2010, 8, 1849-1860.

[10] M. Gómez-García, J. M. Benito, A. P. Butera, C. Ortiz Mellet, J. M. García Fernández, J. L. Jiménez Blanco, J. Org. Chem. 2012, 77, 1273-1288.

[11] D. Ponader, P. Maffre, J. Aretz, D. Pussak, N. M. Ninnemann, S Schmidt, P. H. Seeberger, C. Rademacher, G. U. Nienhaus, L. Hartmann, J. Am. Chem. Soc. 2014, 136, 2008-2016.

[12] L. Otten, M. I. Gibson, RSC Adv. 2015, 5, 53911-53914.

[13] For reviews dealing with the heterocluster effect, see: a) J. L. Jiménez Blanco, C. Ortiz Mellet, J. M. García Fernández, Chem. Soc. Rev. 2013, 42, 4532-4542; b) C. Müller, G. Despras, T. K. Lindhorst, Chem. Soc Rev. 2016, 45, 3275-3302.

[14] For selected recent references on the synthesis of heteroglycoclusters see: a) G. C. Daskhan, C. Pifferi, O. Renaudet, Chem. Open 2016, 5, 477-484; b) S. P. Vincent, K. Buffet, I. Nierengarten, A. Imberty, J.-F. Nierengarten, Chem. Eur. J. 2016, 22, 88-92; c) L. Xue, X. Xiong, Ku. Chen, Y. Luan, G. Chen, H. Chen, Polym. Chem., 2016, 7, 4263-4271; d) S. Jiang, S. Niu, Z.-H. Zhao, Z.-J. Li, Q. Li, Carbohydr. Res. 2015, 414, 39-45; e) A. Meyer, M. Noël, J.-J. Vasseur, F. Morvan, Eur. J. Org. Chem. 2015, 921-2927; f) B. Thomas, M. Fiore, G. C. Daskhan, N. Spinelli, O. Renaudet, Chem. Commun. 2015, 51, 5436-5439; g) M. Fiore, G. C. Daskhan, B. Thomas O. Renaudet, Beilstein J. Org. Chem. 2014, 10, 1557-1563; h) B. Thomas, M. Fiore, I. Bossu, P Dumy, O. Renaudet, Beilstein J. Org. Chem. 2012, 8, 421-427; i) M. Karskela, M. von Usedom, P. Virta, H. Lönnberg, Eur. J. Org. Chem. 2012, 6594-6605.

[15] P. Compain, C. Decroocq, J. lehl, M. Holler, D. Hazelard, T. Mena Barragan, C. Ortiz Mellet, J. F. Nierengarten, Angew. Chem. Int. Ed. 2010, 49, 5753-5756.

[16] C. Decroocq, D. Rodriguez-Lucena, V. Russo, T. Mena Barragan, C Ortiz Mellet, P. Compain, Chem. Eur. J. 2011, 17, 13825-13831.

[17] R. Rísquez-Cuadro, J. M. García Fernández, J. F. Nierengarten, C Ortiz Mellet, Chem. Eur. J. 2013, 19, 16791-16803.

[18] C. Decroocq, A. Joosten, R. Sergent, T. Mena Barragán, C. Ortiz Mellet, P. Compain, ChemBioChem 2013, 14, 2038-2049.

[19] A. Joosten, J. P. Schneider, M. L. Lepage, C. Tarnus, A. Bodlenner, P. Compain, Eur. J. Org. Chem. 2014, 1866-1872.

[20] C. Bonduelle, J. Huang, T. Mena-Barragan, C. Ortiz Mellet, C Decroocq, E. Etame, A. Heise, P. Compain, S. Lecommandoux, Chem Commun. 2014, 50, 3350-3352.

[21] a) M. Durka, K. Buffet, J. lehl, M. Holler, J. F. Nierengarten, S. P. Vincent, Chem. Eur. J. 2012, 18, 641-651; b) A. Tikad, H. Fu, C. M 
Sevrain, S.Laurent, J.-F. Nierengarten, S. P. Vincent, Chem. Eur. J. 2016, 22, 13147-13155.

[22] For reviews, see: a) P. Compain, A. Bodlenner, ChemBioChem 2014 15, 1239-1251; b) S. G. Gouin, Chem. Eur. J. 2014, 20, 11616-11628 c) N. Kanfar, E. Bartolami, R. Zelli, A. Marra, J.-Y. Winum, S. Ulrich, P. Dumy, Org. Biomol. Chem. 2015, 13, 9894-9906; d) R. Zelli, J.-F. Longevial, P. Dumy, A. Marra, New J. Chem. 2015, 39, 5050-5074; e) C. Matassini, C. Parmeggian, F. Cardona, A. Goti, Tetrahedron Lett 2016, 57, 5407-5415

[23] For selected recent references on the synthesis and evaluation of multivalent glycosidase inhibitors, see: a) M. L. Lepage, J. P. Schneider A. Bodlenner, A. Meli, F. De Riccardis, M. Schmitt, C. Tarnus, N.-T. Nguyen-Huynh, Y.-N. Francois, E. Leize-Wagner, C. Birck, A. CousidoSiah, A. Podjarny, I. Izzo, P. Compain Chem. Eur. J. 2016, 22, 51515155 ; b) E. Laigre, D. Hazelard, J. Casas, J. Serra-Vinardell, H. Michelakakis, I. Mavridou, J. M. F. G. Aerts, A. Delgado, P. Compain, Carbohydr. Res. 2016, 429, 98-104; c) T. Hurtaux, G. Sfihi-Loualia, Y Brissonnet, J. Bouckaert, J.-M. Mallet, B. Sendid, F. Delplace, E. Fabre, S. G. Gouin, Y. Guerardel, Carbohydr. Res. 2016, 429, 123-127; d) F. Stauffert, A. Bodlenner, T. M. N. Trinh, M. I. García-Moreno, C. Ortiz Mellet, J.-F. Nierengarten, P. Compain, New J. Chem. 2016, 40, 7421 7430; e) R. Zelli, E. Bartolami, J.-F. Longevial, Y. Bessin, P. Dumy, A. Marra, S. Ulrich, RSC Adv. 2016, 6, 2210-2216.

[24] A. Siriwardena, M. Khanal, A. Barras, O. Bande, T. Mena-Barragan, C. Ortiz Mellet, J. M. García Fernández, R. Boukherroub, S. Szunerits RSC Adv. 2015, 5, 100568-100578.

[25] M. Abellán Flos, M. I. García Moreno, C. Ortiz Mellet, J. M. García Fernández, J.-F. Nierengarten, S. P. Vincent, Chem. Eur. J. 2016, 22 11450-11460

[26] $\mathrm{sp}^{2}$-Iminosugars are carbohydrate mimics in which the endocyclic oxygen typical of monosaccharides has been replaced by a pseudoamide-type nitrogen atom with a high $\mathrm{sp}^{2}$-hybridation character. For selected recent references, see: a) E. M. Sánchez-Fernández, J. M. García Fernández, C. Ortiz Mellet, Chem. Commun. 2016, 52, 54975515; b) T. Mena-Barragán, A. Narita, D. Matias, G. Tiscornia, E. Nanba, K. Ohno, Y. Suzuki, K. Higaki, J. M. García Fernández, C. Ortiz Mellet, Angew. Chem. Int. Ed. 2015, 54, 11696-11700; c) M. de La Mata, D. Cotán, M. Oropesa-Yvila, J. Garrido-Maraver, M. D. Cordero, M. V. Paz, A. D. Pavun, E. Alcocer-Gómez, I. de Lavera, P. YbotGonzález, A. P. Zaderenko, C. Ortiz Mellet, J. M. García Fernández, J A. Sánchez-Alcázar, Sci. Rep. 2015, 5, 10903; d) M. I. García-Moreno, M. de la Mata, E. M. Sánchez-Fernández, J. M. Benito, A. DíazQuintana, S. Fustero, E. Nanba, K. Higaki, J. A. Sánchez-Alcázar, J. M. García Fernández, C. Ortiz Mellet, J. Med. Chem. 2017, in press, DOI: 10.1021/acs.jmedchem.6b01550.

[27] A. Martínez, C. Ortiz Mellet, J. M. García Fernández, Chem. Soc. Rev. 2013, 42, 4746-4773.

[28] a) T. K. Lindhorst, S. Kötter, J. Kubisch, U. Krallmann-Wenzel, S. Ehlers, V. Kren, Eur. J. Org. Chem. 1998, 1669-1674 a) M. FrançoisHeude, A. Méndez-Ardoy, P. L. V. Cendret, R. Daniellou, C. Ortiz Mellet, J. M. García Fernández, V. Moreau, F. Djedaïni-Pilard, Chem. Eur. J. 2015, 21, 1978-1991; b) J. J. Cid Martín, M. Assali, E. Fernández-García, V. Valdivia, E. M. Sánchez-Fernández, J. M. García Fernandez, R. Wellinger, I. Fernandez, N. Khiar, J. Mater. Chem. B 2016, 4, 2028-2037.

[29] M. Almant, A. Mastouri, L. Gallego-Yerga, J. M. Garcia Fernandez, C. Ortiz Mellet, J. Kovensky, S. Morandat, K. El Kirat, S. G. Gouin, Chem. Eur. J. 2013, 19, 729-738.

[30] a) S.-H. Kim, T. Hoshiba, T. Akaike, Biomaterials 2004, 25, 1813-1823; b) E. M. Aguilar Moncayo, N. Guilloteau, C. Bienvenu, J. L. Jiménez Blanco, C. Di Giorgio, P. Vierling, J. M. Benito, C. Ortiz Mellet, J. M. García Fernández, New J. Chem. 2014, 38, 5215-5225.

[31] P. N. Kanellopoulos, K. Pavlou, A. Perrakis, B. Agianian, C. E. Vorgias, C. Mavrommatis, M. Soufi, P. A. Tucker, S. J. Hamodrakas, J. Struct Biol. 1996, 116, 345-355.
[32] R. Banerjee, K. Das, R. Ravishankar, K. Suguna, A. Surulia, M. Vijayan, J. Mol. Biol. 1996, 259, 281-296.

[33] J. B. Corbell, J. J. Lundquist, E. J. Toone, Tetrahedron: Asymmetry 2000, 11, 95-111.

[34] B. Trastoy, D. A. Bonsor, M. E. Pérez-Ojeda, M. L. Jimeno, A. MéndezArdoy, J. M. García Fernández, E. J. Sundberg, J. L. Chiara, Adv. Funct. Mater. 2012, 22, 3191-3201.

[35] a) K. Sasaki, Y. Nishida, T. Tsurumi, H. Uzawa, H. Kondo, K. Kobayashi, Angew. Chem., Int. Ed. 2002, 41, 4463-4467; b) Y. Nishida, H. Dohi and K. Kobayashi, Trends Glycosci. Glycotechnol. 2005, 17, 59-69.

[36] a) V. M. Díaz Pérez, M. I. García-Moreno, C. Ortiz Mellet, J. Fuentes, J. C. Díaz Arribas, F. J. Cañada, J. M. García Fernández, J. Org. Chem. 2000, 65, 136-143; b) J. L. Jiménez Blanco, V. M. Díaz Pérez, C. Ortiz Mellet, F. Fuentes, J. M. García Fernández, J. C. Díaz Arribas, F. J. Cañada, Chem. Commun. 1997, 1969-1970.

[37] E. M. Sánchez-Fernández, R. Rísquez-Cuadro, C. Ortiz Mellet, J. M. García Fernández, P. M. Nieto-Mesa, J. Angulo-Álvarez, Chem. Eur. J. 2012, 18, 8527-8539.

[38] E. M. Sánchez-Fernández, R. Rísquez-Cuadro, M. Aguilar-Moncayo, M. I. García-Moreno, C. Ortiz Mellet, J. M. García Fernández, Org. Lett. 2009, 11, 3306-3309.

[39] K. Dohi, J. Isoyama-Tanaka, R. Misaki, K. Fujiyama, Biochimie 2011, 93, 766-771. 


\section{FULL PAPER}

Different selectivity patterns towards enzymes and lectins can be elicited by (hetero)multivalent displays of sugar and glycomimetic motifs. The binding modes at play reveal analogies between the (hetero)cluster effect and (hetero)multivalent enzyme inhibition that underline the need of a reformulation of the multivalent effect.

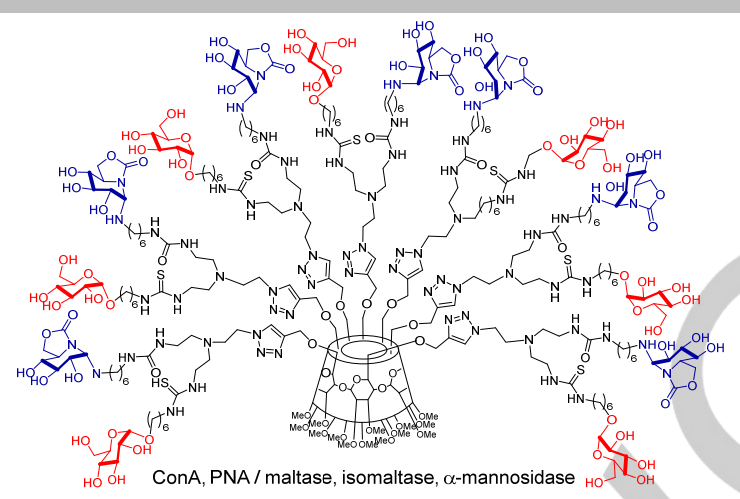

M. I. García-Moreno, F. OrtegaCaballero, ${ }^{[a]}$ R. Rísquez-Cuadro

C. Ortiz Mellet, ${ }^{\star}$ J. M. García

Fernández

Page No. - Page No.

The Impact of

Heteromultivalency in Lectin

Recognition and Glycosidase Inhibition: An Integrated

Mechanistic Study 